\title{
Trapped Microstrip-Ridge Gap Waveguide for Standalone Millimeter Wave Structures
}

\author{
Amir Arayeshnia ${ }^{1}$, Ali Araghi ${ }^{2}$, Mohsen $\mathrm{Khalily}^{2}$, Pei Xiao ${ }^{2}$, Rahim Tafazolli \\ ${ }^{1}$ Faculty of Technical and Engineering, Imam Khomeini International University (IKIU), Qazvin, Iran, \\ a.arayeshnia@edu.ikiu.ac.ir \\ ${ }^{2}$ Institute for Communication Systems (ICS), Home of the 5G Innovation Centre (5GIC), University of Surrey, Guildford, UK \\ \{a.araghi, m.khalily, p.xiao, r.tafazolli\}@surrey.ac.uk
}

\begin{abstract}
This paper presents a novel design of trapped microstrip-ridge gap waveguide by using partially filled air gaps in a conventional microstrip-ridge gap waveguide. The proposed method offers an applicable solution to obviate frustrating assembly processes for standalone high-frequency circuits employing the low temperature co-fired ceramics technology which supports buried cavities. To show the practicality of the proposed approach, propagation characteristics of both trapped microstrip and microstrip-ridge gap waveguide are compared first. Then, a right-angle bend is introduced, followed by designing a power divider. These components are used to feed a linear 4-element array antenna. The bandwidth of the proposed array is $13 \mathrm{GHz}$ from $64 \sim 76$ $\mathrm{GHz}$ and provides the realized gain of over $10 \mathrm{dBi}$ and the total efficiency of about $80 \%$ throughout the operational band. The antenna is an appropriate candidate for upper bands of WiGig (63.72 70.2) and FCC-approved $70 \mathrm{GHz}$ band $(71 \sim 76 \mathrm{GHz})$ applications.
\end{abstract}

Index Terms - Gap waveguide, trapped inverted microstrip line, millimeter wave, $70 \mathrm{GHz}$.

\section{INTRODUCTION}

Waveguides can be considered as an ideal transmission line because of their low loss and high power-handling capability. However, manufacturing waveguides in millimeter or sub-millimeter waves is a mechanical challenge. Recently, gap waveguide technology has been introduced as an effective solution for controlling the electromagnetic fields between metallic parallel plates [1-2]. So far, four basic deviations of the gap waveguide technology have been discussed: groove, ridge, microstrip, and microstrip-ridge. The first two configurations take advantage of a full metallic structure and consequently a low-loss propagation [3-4]. The microstrip gap waveguides are formed by placing a printed strip line between the artificial magnetic conductor (AMC) and the perfect electric conductor (PEC) plates [5]. In the case of microstripridge gap waveguide (M-RGW), the AMC plate and transmission line are implemented using the printed circuit board (PCB) technology [6]. However, it is imperative to mention that all the abovementioned types of gap waveguide technology need complementary assemblyprocesses/structures to hold the PEC plate at a given distance above the AMC plate.

To address this issue, we proposed the trapped microstripridge gap waveguide (TM-RGW) configuration which can be realized using low temperature co-fired ceramics (LTCC) technology supporting buried cavities. The proposed structure contributes to the realization of standalone millimeter-wave (mmWave) circuits and antennas.

The geometry of the TP-RGW is introduced in section II, while the analysis and discussion are presented in section III. To evaluate TM-RGW's capability of realizing standalone structures, a linear corporate fed four-element array antenna is proposed and studied in section IV.

\section{TRAPPED MICROSTRIP-RIDGE GAP WAVEGUIDE CONFIGURATION}

The schematic of the inverted microstrip line is illustrated in Fig. 1 (a). The main difference between conventional and inverted microstrip lines is the location of the ground plane; in the former, it is placed on one side of the substrate while in the latter, the ground plane is located at a specified air distance. In this case, the substrate merely supports the conductive strip and a relatively high portion of fields are concentrated in the air gap between the strip and the ground plane. This configuration reduces power dissipation and makes the inverted microstrip an appropriate choice to be applied in the mmWave band [7].

The trapped inverted microstrip (TIM) structure is similar to the inverted microstrip from almost all aspects, except that the ground plane forms a shallow trench which effectively isolates strip line from three directions, as shown in Fig. 1 (b). This configuration has better isolation from the surrounding environment in comparison with the inverted microstrip, appropriates for mm-wave applications [8].

M-RGW geometry is depicted in Fig. 1 (c). The conductive strip (at the middle of the figure) is connected to

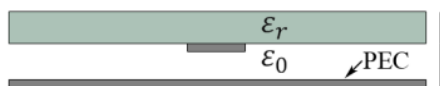

(a)

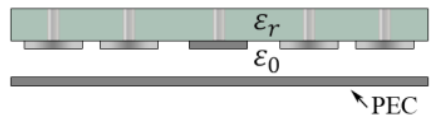

(c)

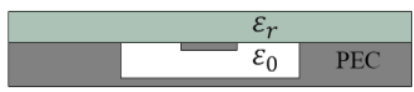

(b)

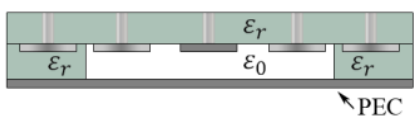

(d)
Fig. 1. The schematic of (a) inverted microstrip, (b) trapped microstrip line, (c) microstrip-edge gap waveguide, and (d) the proposed trapped microstripridge gap waveguide. 
the ground plane through semi-periodic vias, surrounded by rows of mushroom structure. The periodic arrangement of mushroom structures in the substrate creates an AMC layer and suppresses wave propagation in the air gap between the PCB layer (substrate and mushroom structures) and conductive plane (PEC). In other words, the waves will mostly propagate along the conductive strip. It must be noted that the air distance between AMC and PEC layers should be smaller than a quarter wavelength [1]. In general, the isolation performance of the M-RGW is relatively similar to the trapped inverted microstrip, but with a different mechanism. However, despite the TIM, the M-RGW needs additional structures and assembly processes to keep the air distance between AMC and PEC layers.

The schematic of the proposed TM-RGW is presented in Fig. 1 (d). As depicted, the air gap between AMC and PEC layers is partially filled, in a way to form an air duct around the inverted microstrip line. While this configuration reduces the assembly challenges, it does not dramatically change the transmission line performance because of the EM-field concentration around the inverted microstrip. Also, the reflection at the boundary of air and dielectric sidewalls contributes to further confining of electromagnetic waves within the air duct.

\section{TRAPPED MICROSTRIP-RIDGE GAP WAVEGUIDE ANALYSIS}

Mushroom structures can be employed to realize the AMC plate. In this case, the stop-band spectrum is related to the thickness and permittivity coefficient of the substrate, air gap's height, pad diameter, and the period length. In this regard, the Eigenmode solver of CST microwave studio commercial package is used to design an appropriate mushroom structure to create a stop-band spectrum around $70 \mathrm{GHz}$ using a substrate with $\varepsilon_{\mathrm{r}}=5.9$ and $\tan \delta=0.002$.

The Geometry of the mushroom unit cell and corresponding dispersion diagrams for $\mathrm{w} 1=0.575 \mathrm{~mm}$, $\mathrm{g}=0.127 \mathrm{~mm}, \mathrm{~h} 1=0.254 \mathrm{~mm}, \mathrm{dp}=0.42 \mathrm{~mm}, \mathrm{dv}=0.12 \mathrm{~mm}$ are presented in Fig. 2. As can be seen, there is no propagated mode in the 58 88 GHz frequency band. Fig. 3 shows the geometry of M-RGW transmission line unit cell and related dispersion diagrams. The width of the inverted microstrip line

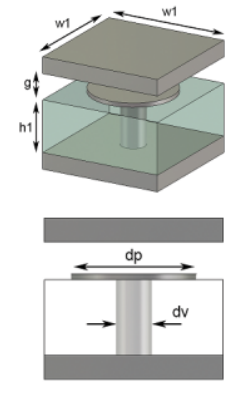

(a)

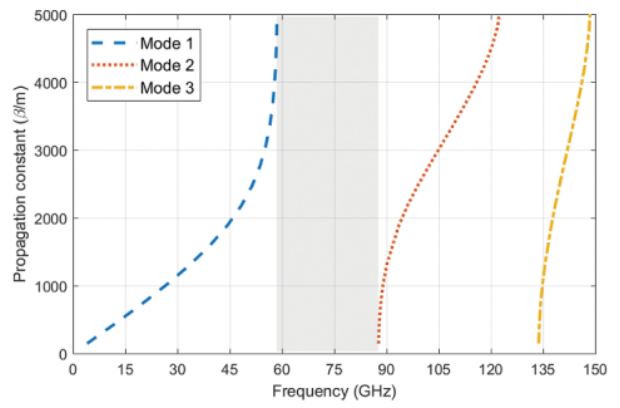

(b)
Fig. 2. (a) The geometry of mushroom unit cell and (b) the corresponding dispersion diagram. $\mathrm{w} 1=0.575 \mathrm{~mm}, \mathrm{~g}=0.127 \mathrm{~mm}, \mathrm{hl}=0.254 \mathrm{~mm}, \mathrm{dp}=0.42 \mathrm{~mm}$, $\mathrm{dv}=0.12 \mathrm{~mm}$.

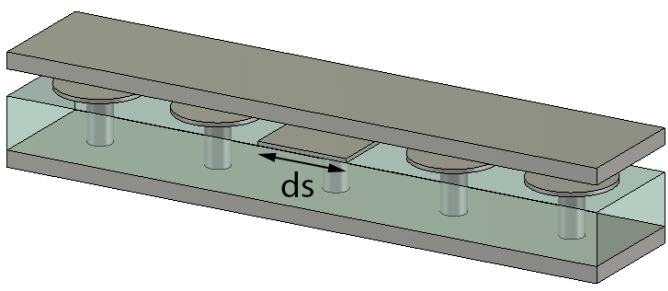

(a)

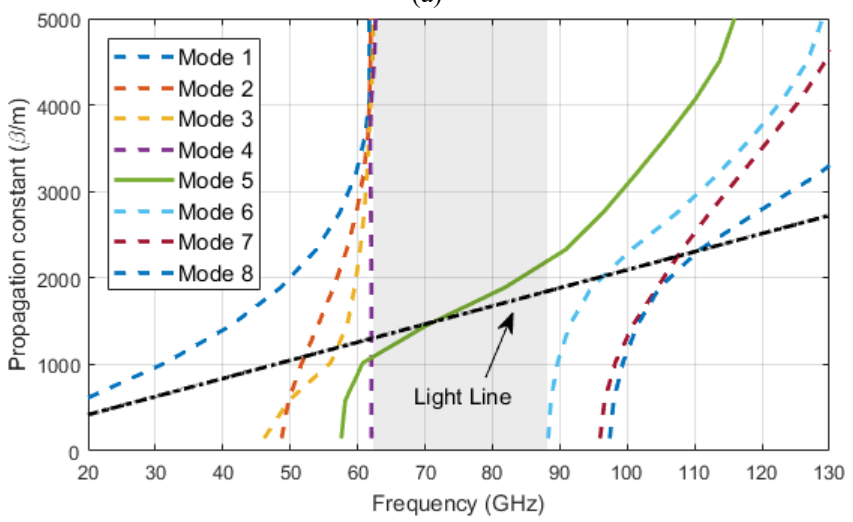

(b)

Fig. 3. (a) The geometry of mushroom unit cell and (b) the corresponding dispersion diagram.

has set $\mathrm{ds}=0.45 \mathrm{~mm}$. It is observed that only one mode can propagate along the transmission line in $62 \sim 88 \mathrm{GHz}$ frequency band.

As it is mentioned before, the electrical field distribution in the M-RGW transmission line is mainly concentrated around the inverted microstrip. This suggests that the air gap above adjacent mushroom cells can be filled with a dielectric material, creating a duct around the inverted microstrip line without changing the propagation characteristic significantly. This ducting can be implemented using LTCC technology which supports buried cavities, mitigates assembly processes for PEC plate suspension over the AMC plate. The width of the dielectric duct can be determined by considering the fabrication limitations as well as intended characteristics such as dissipation and single-mode bandwidth. In the following the duct width is set to $1.8 \mathrm{~mm}$.

\section{A. Propagation Characteristics}

The dispersion diagram of the TM-RGW for both trapped configuration and dielectric-loaded mushroom structures are presented in Fig. 4. It is found that the dielectric loading of mushroom unit cells leads to the shift of stop-band to the lower frequencies. However, as expected, due to the low concentration of electrical fields in the farther mushroom rows, the single-mode spectrum remains almost unchanged.

The electrical field distribution at the cross-section of both M-RGW and TM-RGW transmission lines is presented in Fig. 5. The results indicate that most electric fields are concentrated around the conductive strip in both cases. Furthermore, the discrepancy between air and dielectric sidewalls improves the isolation and confinement of electric fields in the air duct. 
The operating frequency of the proposed trapped configuration is about $62 \sim 82 \mathrm{GHz}$ which is the overlapping range of TM-RGW single-mode band and conventional and dielectric-loaded mushroom unit cell stop-bands. In this range, waves can propagate only along the trapped inverted microstrip line and fade at other directions.

To obtain the proposed TM-RGW line loss, the cut-back technique is employed [9]. In this method, the line loss can be computed by simulating the transmitted power at two points with a specified distance through the transmission line. The advantage of this method is that due to the constant excitation conditions in both simulations, the input port mismatch does not cause an error in the line loss calculation. The calculated line loss of M-RGW and TM-RGW at $70 \mathrm{GHz}$ is 0.0890 and $0.0845 \mathrm{~dB} / \mathrm{cm}$, respectively. The simulated results indicate that the air duct contributes to a slight improvement of propagation loss with better isolation as well as the inherent advantage of a simpler assembly process.

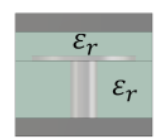

(a)

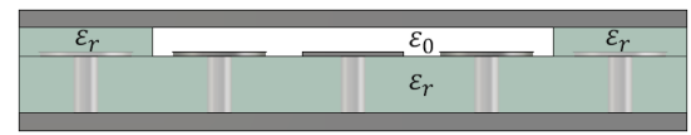

(b)

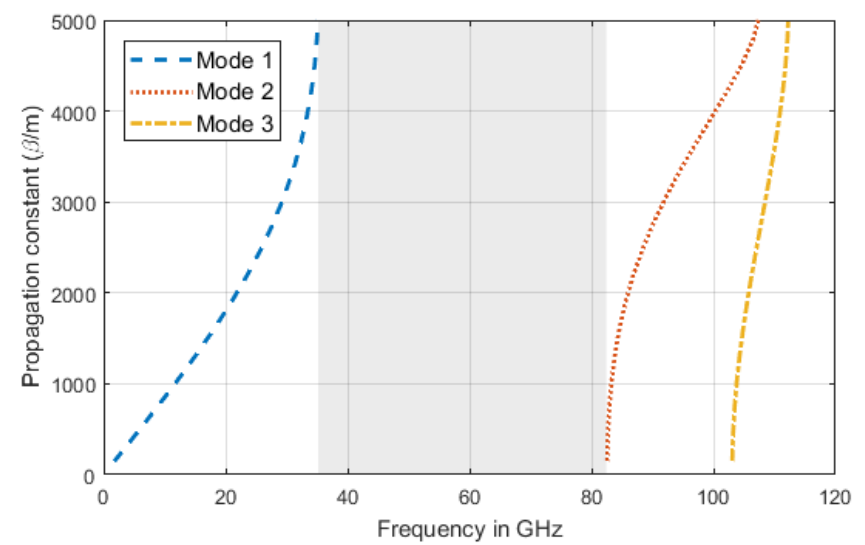

(c)

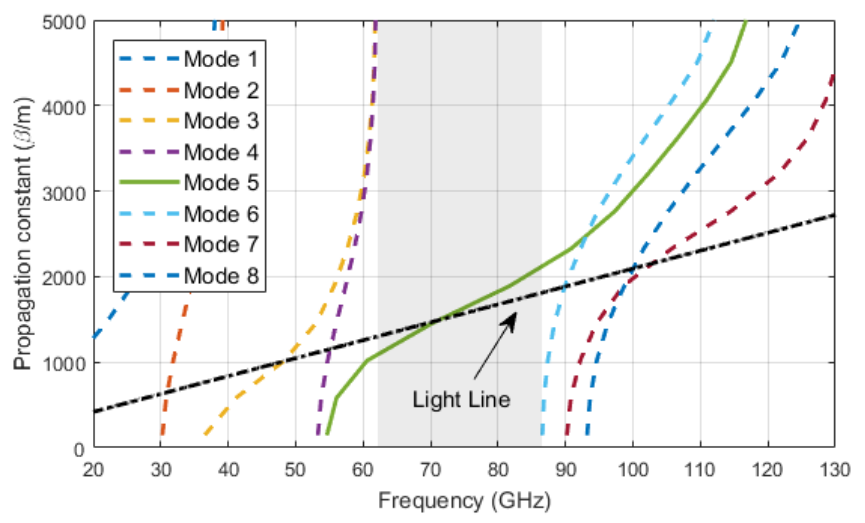

(d)

Fig. 4. The geometry of (a) the dielectric-loaded mushroom and (b) TMRGW unit cells. the dispersion diagrams of (c) dielectric-loaded mushroom and (d) TM-RGW unit cells.
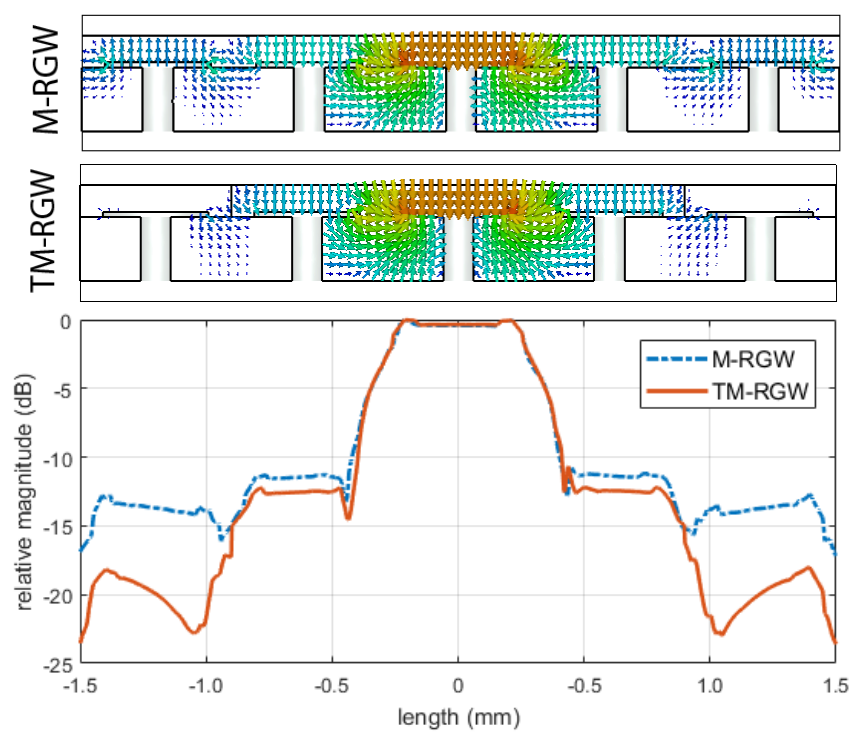

Fig. 5. Electrical field distribution at the cross-section of the TM-RGW transmission line.

\section{ANTENNA ARRAY DESIGN}

In order to evaluate the integration capability of the TMRGW structure, a linear antenna array is designed and simulated. The right-angle bend and power divider are essential components for the implementation of a corporate fed array. The conventional mitered geometry for M-RGW right angle bend suffers from severe mismatching. A reported solution to this issue is based on rearranging mushroom structures around the mitered bending area [6].

\section{A. Bend and Divider}

The proposed geometries for the right-angle bend is formed by cutting out a radial stub from the radial bend section without disturbing mushroom's periodicity, as shown in Fig. 6 (a). The geometry of the proposed power divider is depicted in Fig. 6 (b), which contains a quarter-wave transformer and two radial-notched bend sections. The reflection and transmission coefficients for both components are given in Fig. 6 (c). As can be seen, an appropriate matching is achieved over $62 \sim 85 \mathrm{GHz}$ spectrum.

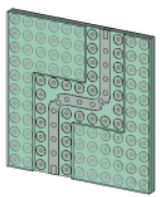

(a)

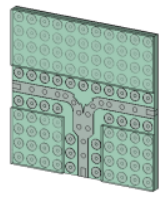

(b)

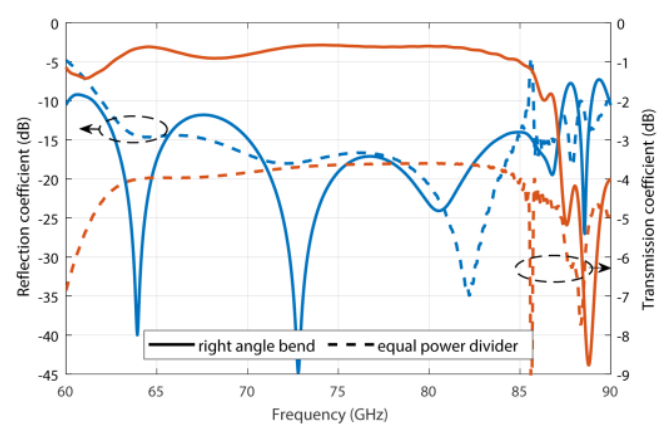

(c)
Fig. 6. The proposed (a) right angle bend and (b) equal power divider, and (c) the corresponding reflection and transmission coefficients. The upper PEC plate is not shown. 


\section{B. Radiating Aperture}

A miniaturized low-profile antenna based on quasicomposite right/left-hand metamaterials is employed as the radiating element [10]. A single antenna element consists of a $4 \times 4$ printed metamaterial array fed by the slot-coupled method. The resonance frequencies of $\mathrm{TM}_{10}$ and antiphase $\mathrm{TM}_{20}$ are designed to be about $60 \mathrm{GHz}$ and $70 \mathrm{GHz}$, respectively.

Figure 7 shows the exploded and side view of the fourelement linear antenna array fed by a corporate feeding consisted of right-angle bend and the designed power divider. It must be noted that the antenna substrate thickness can be reached by the aggregation of standard 5 and 2 mill thickness substrates ( $\mathrm{h} 1=10 \mathrm{mil}, \mathrm{g}=5 \mathrm{mil}, \mathrm{h} 2=9 \mathrm{mil}$ ).

The E-fields distribution within the structure at $70 \mathrm{GHz}$ is presented in Fig. 8. As shown, E-fields are effectively guided across the trapped inverted microstrip line at the observed frequency.

The simulated reflection coefficient of the array antenna is shown in Fig. 9. The impedance bandwidth is about 64 76 $\mathrm{GHz}$ which is within the TM-RGW operational band (i.e. $62 \sim 82 \mathrm{GHz}$ ). The antenna is applicable for upper bands of WiGig (63.72 70.2) and FCC-approved $70 \mathrm{GHz}$ band (71 76 GHz) applications.

Figure 10 shows the realized gain and total efficiency of the array antenna over the interested spectrum. The realized gain exceeds $10 \mathrm{dBi}$ overall spectrum with the maximum value of $13.5 \mathrm{dBi}$, while the total efficiency fluctuates around $80 \%$.

The radiation patterns in E- and H-planes in different frequencies are also illustrated in Fig. 11. It is observed that the antenna provides a stable unidirectional pattern in the boresight direction throughout the operational bandwidth.

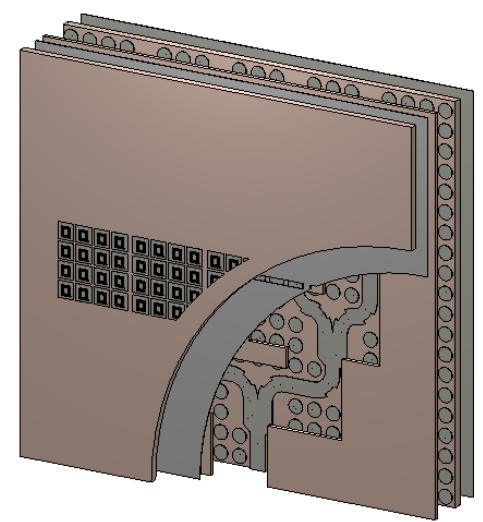

(a)

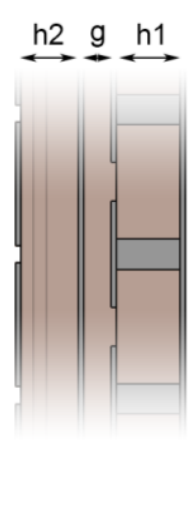

(b)
Fig. 7. (a) The exploded and (b) side view of the proposed 4 element linear array antenna. $\mathrm{h} 1=0.254 \mathrm{~mm}, \mathrm{~g}=0.127 \mathrm{~mm}, \mathrm{~h} 2=0.2286 \mathrm{~mm}$.
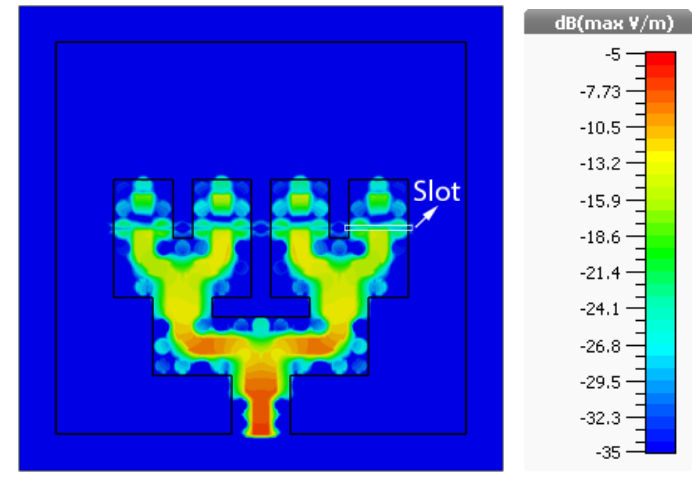

Fig. 8. Electrical field propagation through the corporate-fed network at 70 $\mathrm{GHz}$.

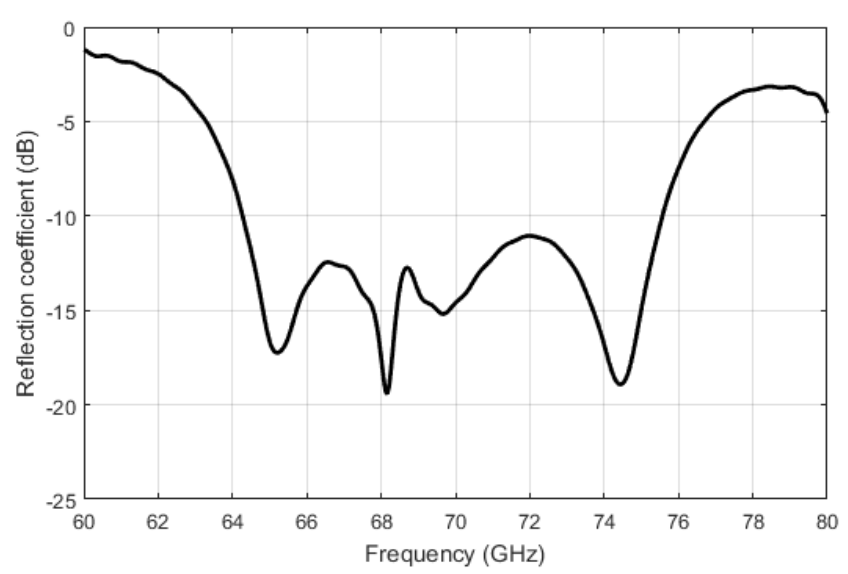

Fig. 9. The simulated reflection coefficient of the proposed array antenna.

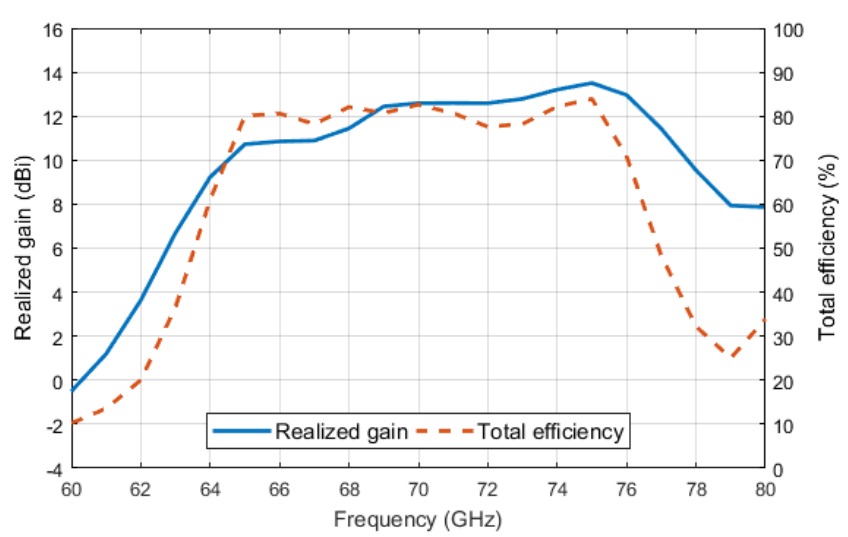

Fig. 10. The simulated realized gain and total efficiency of the proposed array antenna. 


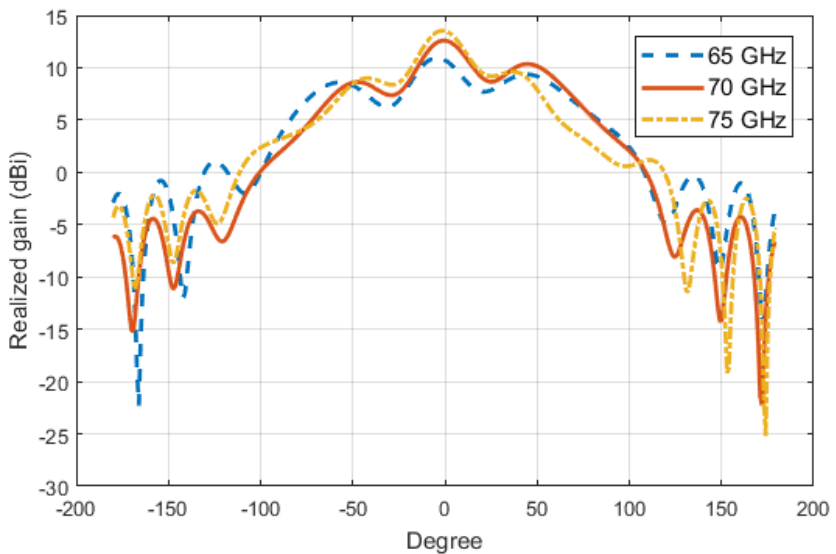

(a)

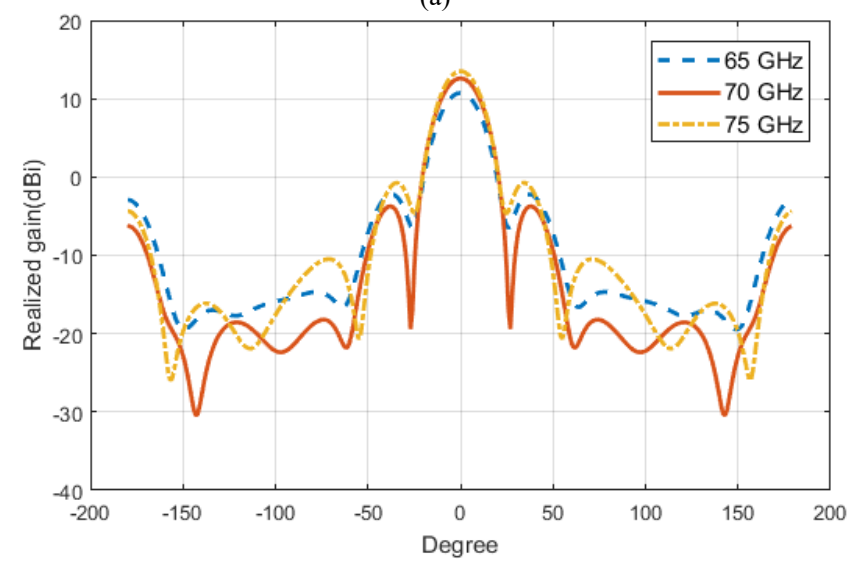

(b)

Fig. 11. The simulated radiation patterns of the proposed array antenna in (a) $\mathrm{H}$ - and (b) E-plane.

\section{CONCLUSION}

The TM-RGW configuration using LTCC technology has been studied. In the proposed design, the air gap between PEC and AMC plates is partially filled in a way that the inverted microstrip line is trapped between two dielectric walls. This configuration obviates the further assembly processes for suspension of PEC plate over the AMC plate which is essential for gap waveguides. The propagation characteristics of the proposed TM-RGW and conventional M-RGW were also compared. It was found that both configurations have a relatively similar applicable spectrum. A new right angle bend and equal power divider without disturbing the AMC mimicking mushroom cells are also introduced. The results show that their reflection coefficients are below $-10 \mathrm{~dB}$ over $62 \sim 85 \mathrm{GHz}$ spectrum. Finally, a corporate fed 4-element linear array antenna was designed and simulated results were discussed. The results indicate that the proposed array antenna has a realized gain of more than $10 \mathrm{dBi}$ with the maximum value of $13.5 \mathrm{dBi}$ and total efficiency of about $80 \%$ throughout the impedance bandwidth of $64 \sim 76 \mathrm{GHz}$. The reported total efficiency demonstrates the potential of the TM-RGW configuration for the realization of standalone high-efficient mmWave circuits and antennas.

\section{REFERENCES}

[1] P. Kildal, E. Alfonso, A. Valero-Nogueira and E. Rajo-Iglesias, "Local Metamaterial-Based Waveguides in Gaps Between Parallel Metal Plates", IEEE Antennas and Wireless Propagation Letters, vol. 8, pp. 84-87, 2009.

[2] P. Kildal, "Three metamaterial-based gap waveguides between parallel metal plates for mm/submm waves," 2009 3rd European Conference on Antennas and Propagation, Berlin, 2009, pp. 28-32.

[3] P. -. Kildal, A. U. Zaman, E. Rajo-Iglesias, E. Alfonso and A. ValeroNogueira, "Design and experimental verification of ridge gap waveguide in bed of nails for parallel-plate mode suppression," in IET Microwaves, Antennas \& Propagation, vol. 5, no. 3, pp. 262-270, 21 Feb. 2011.

[4] E. Rajo-Iglesias and P. Kildal, "Groove gap waveguide: A rectangular waveguide between contactless metal plates enabled by parallel-plate cut-off," Proceedings of the Fourth European Conference on Antennas and Propagation, Barcelona, 2010, pp. 1-4.

[5] A. A. Brazález, E. Rajo-Iglesias, J. L. Vázquez-Roy, A. Vosoogh and P. Kildal, "Design and Validation of Microstrip Gap Waveguides and Their Transitions to Rectangular Waveguide, for Millimeter-Wave Applications," in IEEE Transactions on Microwave Theory and Techniques, vol. 63, no. 12, pp. 4035-4050, Dec. 2015.

[6] H. Raza, J. Yang, P. Kildal and E. Alfonso Alos, "Microstrip-Ridge Gap Waveguide-Study of Losses, Bends, and Transition to WR-15", IEEE Transactions on Microwave Theory and Techniques, vol. 62, no. 9, pp. 1943-1952, 2014.

[7] T. Edwards and M. Steer, Foundations for Microstrip Circuit Design, 4th Edition, John Wiley \& Sons, 2016.

[8] R. Bera and R. Wallace, "Trapped Inverted Microstrip (TIM) Circuits for Combining the Outputs of High-Power IMPATT Oscillators", MTT-S International Microwave Symposium Digest.

[9] R. Hui and M. O'Sullivan, Fiber optic measurement techniques. Amsterdam: Elsevier/Academic Press, 2009, pp. 382-384.

[10] A. Arayeshnia, A. Bayat, M. Keshtkar-Bagheri and S. Jarchi, "Miniaturized low-profile antenna based on uniplanar quasi-composite right/left-handed metamaterial", International Journal of $R F$ and Microwave Computer-Aided Engineering, p. e21888, 2019. 